\title{
Frequency of Infectious Mortality at the End of Induction Chemotherapy in Acute Lymphoblastic Leukemia and Lymphoma Patients: Findings From a Tertiary Care Cancer Center
}

\author{
Rabia Wali ${ }^{1}$, Sadia Anjum ${ }^{1}$, Asim Amjad ${ }^{2}$, Najma Shaheen ${ }^{1}$, Saadiya Javed Khan ${ }^{3}$ \\ 1. Paediatric Oncology, Shaukat Khanum Memorial Cancer Hospital and Research Centre, Lahore, PAK 2. Paediatrics, \\ Mayo Hospital, Lahore, PAK 3. Paediatrics and Child Health, King Faisal Specialist Hospital and Research Centre, \\ Riyadh, SAU
}

Corresponding author: Rabia Wali, rwali@skm.org.pk

\section{Abstract \\ Background and objective}

In low- and low-to-middle-income countries (LMICs), the incidence of treatment-related mortality (TRM) in patients with acute lymphoblastic leukemia (ALL) and lymphoblastic lymphoma (LBL) is up to $52 \%$. This study aimed to determine the mortality rate at the end of the induction phase of the treatment among patients with ALL and lymphoma at a tertiary care cancer center.

\section{Methods}

This retrospective study analyzed outcomes after induction chemotherapy in pediatric patients with acute leukemia and lymphoma at a tertiary care cancer center from January 2015 to December 2016. Information regarding demographics, clinical characteristics, and laboratory investigations were extracted and reviewed.

\section{Results}

Of the total 160 patients, 110 were males, and the mean age of the sample was $4.6+2.8$ years. B-cell leukemia (pre-B-ALL) was diagnosed in $84 \%(n=134)$, while $10 \%(n=6)$ had acute T-cell leukemia (pre-T-ALL) and $6 \%$ $(\mathrm{n}=10)$ had lymphoma. Sixteen patients $(10 \%)$ died within the defined induction period, with 14 deaths occurring due to infections and two deaths resulting from chemotherapy-related toxicity.

\section{Conclusion}

Based on our findings, there is a significant prospect of mortality from infections during induction chemotherapy in patients with pediatric hematological malignancies.

Review began 01/21/2021 Review ended 01/31/2021 Published 02/07/2021

\section{() Copyright 2021}

Wali et al. This is an open access article distributed under the terms of the Creative Commons Attribution License CC-BY 4.0., which permits unrestricted use, distribution, and reproduction in any medium, provided the original author and source are credited.
Categories: Pediatrics, Oncology, Hematology

Keywords: acute leukemia, induction chemotherapy, lymphoblastic lymphoma, mortality

\section{Introduction}

Acute lymphoblastic leukemia (ALL) and lymphoblastic lymphoma (LBL) are among the most common hematological malignancies among pediatric patients and account for approximately $30 \%$ of all childhood cancers. In the last 30 years, advances made in treatment have led to five-year survival rates of more than $80 \%$ [1]. This has been accomplished through a better understanding of tumor biology at a molecular level, risk assignment, and intensification of chemotherapeutics based on a risk-adapted approach. Furthermore, improvements in supportive care strategies such as early detection and prompt treatment of infections, optimization of blood product administration, and early intensive care (ICU) referrals have contributed to better outcomes [2,3]. Nonetheless, children may still experience extremely toxic drug-induced side effects, which can even result in treatment-related mortality (TRM). The majority of these reactions occur during the initial induction phase of the treatment [4].

The incidence of TRMs due to infections in patients with acute leukemia and lymphoma is about 2-4\% [5]. However, the TRM percentage in low-to-middle-income countries (LMICs) is much higher, and TRM incidence of up to $52 \%$ has been reported [6-8]. This disparity in rates is likely due to infections, suboptimal supportive care, malnutrition, chemotherapy-related toxicity, delay in diagnosis, and abandonment due to social issues [9].

The primary aim of this study was to determine the mortality rate at the end of the induction phase of the treatment in patients with ALL and LBL at a tertiary care cancer center operating in an LMIC. 


\section{Materials And Methods}

We conducted a retrospective study on pediatric ALL and LBL patients treated at the Shaukat Khanum Memorial Cancer Hospital (SKMCH) between January 2015 and December 2016. All patients with leukemia and lymphoma treated with a standard chemotherapeutic regimen were included in the study. However, patients of less than one year of age or those with mature B- and T-cell lymphoma were excluded. The Institutional Review Board at SKMCH approved the study and gave the go-ahead to collect data on patient demographics, clinical features, pathology, imaging, treatment, and outcomes from the hospital's electronic medical records (approval number: EX-01-08-17-02-A1).

The patients were risk-stratified according to the National Cancer Institute (NCI) guidelines and treated based on the United Kingdom Acute Lymphoblastic Leukemia (UKALL) 2013 guidelines [10]. Cranial (CNS) disease was defined as the presence of lymphoblasts on cytospin preparation at the time of diagnostic lumbar puncture. Patients between 1-9.99 years of age, those with a white cell count of less than 50,000, or those with precursor B-cell immunophenotype were stratified as standard risk (SR). On the contrary, patients were categorized as high risk (HR) if they were above 10 years of age, if their white cell count was more than or equal to 50,000, or if they had precursor T-cell immunophenotype and LBL. The SR patients received a three-drug induction for four weeks with dexamethasone $6 \mathrm{mg} / \mathrm{m}^{2}$ for 28 days, vincristine weekly, and PEGylated asparaginase in two doses. The HR patients received a four-drug induction with the addition of daunorubicin weekly to the above-mentioned three-drug regimen. All patients received intrathecal (IT) methotrexate (MTX) on day one and subsequent weekly ITs based on CNS involvement.

All newly diagnosed ALL and LBL patients were admitted to the hospital for the initiation of the treatment. They were managed for tumor lysis prophylaxis for the first 24-48 hours, which was followed by chemotherapy. Bone marrow re-assessment was done on days eight and 15 based on risk stratification and then at end of the induction on day 29 in all patients for disease remission. If patients were clinically well after day four of the induction chemotherapy, they were discharged and then were subsequently seen every week in the outpatient clinic.

While on induction therapy, all patients were advised to come to the emergency room if they had a fever. Fever was defined as a single instance of a temperature of at least $38^{\circ} \mathrm{C}$ within the last one hour or a single spike of $38.3^{\circ} \mathrm{C}[4]$. In the emergency room, all patients with fever underwent a thorough history and examination and had complete blood count, blood cultures, and urine culture or chest imaging, wherever clinically appropriate. Furthermore, a stat dose of broad-spectrum antibiotics was administered. Patients were hospitalized and clinically presumed to have an infection if the absolute neutrophil count was less than 1,000 in the absence of positive cultures.

Diagnosis of infection was validated with microbiologically proven blood cultures, urine cultures, tissue specimens from the chest or fine needle aspirations, or endoscopically from core biopsy of sinuses. Blood cultures were taken on admission and then repeated in cases of new fever, change of antibiotics, and daily in case of persistent fevers. The causes of death were categorized as infection-associated, treatment-related toxicity, or bleeding complications. The duration of the induction was 42 days from the first day of admission including the pre-induction phase, and all deaths that occurred during this period were included in this analysis.

All the collected data were analyzed using Microsoft Excel version 10 (Microsoft Corporation, Redmond, WA) and SPSS Statistics version 19 (IBM, Armonk, NY). Descriptive statistics were applied to calculate the mean and standard deviation. Frequency distribution and percentages were calculated for qualitative variables using a sample t-test. Overall, a p-value of less than 0.05 was considered statistically significant.

\section{Results}

A total of 160 patients were included in this study. The mean age of the sample was $4.6+2.8$ years, and more than $90 \%$ of the patients were less than five years of age. The male-to-female ratio was $2.2: 1$. The most common primary disease was B-cell leukemia (pre-B-ALL; 83.8\%), and most of the participants were categorized as low risk (76.9\%). During the defined induction period, 16 patients (10\%) died (Table 1 ). Among these, 14 (87.5\%) deaths were infection-associated, and two (12.5\%) were from chemotherapyrelated toxicity. Gastrointestinal manifestations were seen in four patients (25\%), while five (32\%) had respiratory symptoms along with positive cultures and seven (43\%) patients had bacteremia.

Infections were microbiologically verified in 12 (85\%) patients. In these 12 patients with a pathologically proven bacterial infection, there were a total of 16 positive cultures: nine positive blood cultures, two tracheal aspirates, two endoscopic sinus biopsies, and one each for peritoneal fluid, CT-guided lung biopsy, and ear swabs were isolated.

Nine cultures (56\%) were positive for gram-negative bacteria (GNB), and among these, five cultures had multidrug-resistant Escherichia coli (MDR-E. coli), three had pan-sensitive E.coli, and one sample came back positive for pseudomonas. Similarly, in five subjects, five (31\%) samples tested positive for fungal infection. 


\section{Cureus}

Three subjects with positive fungal cultures also had positive bacterial cultures. Two tracheal aspirates and a lung nodule grew fungal Aspergillus, and Mucor grew from one endoscopic biopsy and Fusarium from the ear discharge (Table 2).

\begin{tabular}{|c|c|}
\hline Patient parameters & $\mathbf{N}(\%)$, total= \\
\hline \multicolumn{2}{|l|}{ Age } \\
\hline 1-5 years & 147 (92\%) \\
\hline$\geq 5$ years & $13(8 \%)$ \\
\hline \multicolumn{2}{|l|}{ Gender } \\
\hline Male & $110(69 \%)$ \\
\hline Female & $50(31 \%)$ \\
\hline \multicolumn{2}{|l|}{ Primary disease } \\
\hline Pre-B-ALL & $134(84 \%)$ \\
\hline Pre-T-ALL & $16(10 \%)$ \\
\hline T-/B-LBL & $10(6 \%)$ \\
\hline \multicolumn{2}{|l|}{ Risk group } \\
\hline Low risk & 123 (77\%) \\
\hline High risk & $37(23 \%)$ \\
\hline \multicolumn{2}{|l|}{ Infections } \\
\hline Yes & 14 (8.75\%) \\
\hline No & 146 (91.25\%) \\
\hline \multicolumn{2}{|l|}{ utcomes } \\
\hline Alive & $144(90 \%)$ \\
\hline Dead & $16(10 \%)$ \\
\hline
\end{tabular}

\section{TABLE 1: Characteristics of leukemia and lymphoma patients}

B-ALL: B-cell acute lymphoblastic leukemia; T-ALL: T-cell acute lymphoblastic leukemia; LBL: lymphoblastic lymphoma 


\section{Cureus}

\begin{tabular}{|c|c|c|}
\hline Patients ( $(=12)$ & Organisms cultured & Positive cultures/site $(n=16)$ \\
\hline 1 & Aspergillus & Tracheal aspirate \\
\hline 2 & MDR E.coli & Blood CS \\
\hline 3 & MDR E.coli & Blood CS \\
\hline 4 & MDR E.coli & Blood CS \\
\hline 5 & E.coli & Blood CS \\
\hline 6 & E.coli & Peritoneal fluid \\
\hline \multirow[t]{2}{*}{7} & MDR E.coli & Blood CS \\
\hline & Mucor & Endoscopic biopsy from sinus \\
\hline 8 & Fungal hyphae & CT chest nodule biopsy \\
\hline 9 & Pseudomonas & Blood CS \\
\hline \multirow[t]{2}{*}{10} & E.coli & Blood CS \\
\hline & Aspergillus & Tracheal aspirate \\
\hline \multirow[t]{2}{*}{11} & Vancomycin-resistant Enterococci & Endoscopic biopsy from sinus \\
\hline & Fusarium & Ear swab \\
\hline \multirow[t]{2}{*}{12} & MDR E.coli & Blood CS \\
\hline & Vancomycin-resistant Enterococci & Blood CS \\
\hline
\end{tabular}

TABLE 2: Positive cultures from all sites

MDR: multidrug-resistant; E. coli: Escherichia coli

One of the two toxicity deaths was secondary to pancreatitis leading to disseminated intravascular coagulation (DIC). The other was related to vincristine-induced paralytic ileus leading to a mechanical obstruction that resulted in bowel perforation. All deaths occurred between 15 to 24 days of the induction phase. Bone marrow was in remission on day 29 in 136 patients (85\%). Of the 16 subjects who died, bone marrow examination was possible in only two patients, and both were in remission.

\section{Discussion}

Favorable outcomes related to ALL and LBL in LMICs remain low despite improvements in supportive care services. TRM, primarily due to infections, remains one of the significant factors affecting outcomes. Notably, a high number of deaths occurs during the early induction phase. This study aimed to determine the mortality rate at the end of the induction among patients with leukemia and lymphoma at a tertiary care cancer center operating in an LMIC. The incidence of induction mortality in this study was $10 \%$. Of these, $87.5 \%$ of the deaths were related to infections, and $12.7 \%$ were secondary to chemotherapy-related toxicity.

Studies from various LMICs have reported induction death rates ranging from 12.8 to $22.6 \%[11,12]$. In comparison, in prior retrospective analyses from the center where the present study was conducted, the induction death ranged from 24 to $52 \%$ [8,13]. In the present study, TRM was found to be $10 \%$. This improvement in outcome is likely associated with the developments in infrastructural facilities, quality and availability of blood products, and better infection control practices and treatment measures. Nonetheless, this rate is still three to four times higher than the rates reported in high-income countries [14].

In the present study, $87.5 \%$ of the induction deaths were associated with infections, and among 16 positive cultures, $56 \%$ were associated with GNB. These results are similar to those reported by Fadoo et al. [6]. Investigators found that $63.5 \%$ of the 52 samples of blood cultures were significant for GNB. Generally, it appears that there is a low percentage of positive cultures of gram-positive bacteria. The exact reason for this is unknown and beyond the scope of this manuscript. However, it could be secondary to a lack of central line placement in this cohort of patients.

Fungal infections pose a significant threat to children with ALL and lymphomas. Previously, the rate of invasive fungal infections among patients with hematological malignancies was reported to be between 1.3- 
$25 \%[15,16]$. However, in the present study, the fungal infection rate was $31 \%$, and among these, three subjects had concurrent positive cultures for bacteremia. During the induction phase, patients develop neutropenia and are immunocompromised, making them susceptible to bacterial and fungal infections. However, this alone may not explain why nearly one-third of the infections were fungal in origin in the present study. A possible contributing factor could be the onsite construction during the study period. Multiple reports in the literature suggest an association between invasive fungal infections and contaminated ventilation systems, hospital construction, or renovations $[17,18]$.

Seven (44\%) out of the 16 bloodstream infections were MDR E.coli and vancomycin-resistant Enterococci. This high percentage could be due to the particular characteristics of the cohort of patients who are treated at our center. The majority of the patients are referred to from low-income communities, where indiscreet use of antibiotics is common for minor ailments (fever, flu, and respiratory or gastrointestinal symptoms). Furthermore, due to the inherent nature of hematological malignancies, these patients frequently experience fever, which is routinely managed locally with broad-spectrum intravenous antibiotics.

A limitation of this study is that it was a retrospective analysis. Hence, it was impossible to have an equal distribution of subjects with SR and HR, central line access in all subjects, or control for other clinical and management-associated characteristics. Moreover, due to the nature of the research design, there was a lack of randomization and blindness, resulting in a high likelihood of false-positive associations or magnification of positive responses. Nonetheless, data were extracted from an electronic database and verified by reviewing follow-up visits.

\section{Conclusions}

In conclusion, there is a significant prospect of mortality associated with induction chemotherapy among patients with ALL and lymphoma. A significant number of deaths in our study were secondary to infections. Infections remain a major healthcare challenge in LMICs. Raising awareness about the resistance caused by excessive antibiotic usage can further help decrease our mortality rates. Going forward, we intend to examine the prophylactic antibiotic use during induction therapy among our patients in a prospective manner.

\section{Additional Information \\ Disclosures}

Human subjects: Consent was obtained or waived by all participants in this study. Shaukat Khanum Memorial Cancer Hospital Institutional Review Board issued approval EX-01-08-17-02-A1. This study was approved by the Institutional Review Board at the Shaukat Khanum Memorial Cancer Hospital. Animal subjects: All authors have confirmed that this study did not involve animal subjects or tissue. Conflicts of interest: In compliance with the ICMJE uniform disclosure form, all authors declare the following: Payment/services info: All authors have declared that no financial support was received from any organization for the submitted work. Financial relationships: All authors have declared that they have no financial relationships at present or within the previous three years with any organizations that might have an interest in the submitted work. Other relationships: All authors have declared that there are no other relationships or activities that could appear to have influenced the submitted work.

\section{Acknowledgements}

We would like to thank our patients and their families.

\section{References}

1. Hunger SP, Lu X, Devidas M, et al.: Improved survival for children and adolescents with acute lymphoblastic leukemia between 1990 and 2005: a report from the Children's Oncology Group. J Clin Oncol. 2012, 30:16631669. 10.1200/JCO.2011.37.8018

2. Pui CH, Evans WE: Treatment of acute lymphoblastic leukemia . N Engl J Med. 2006, 354:166-178. 10.1056/NEJMra052603

3. Cheng S, Pole JD, Sung L: Early deaths in pediatric acute leukemia: a population-based study . Leuk Lymphoma. 2014, 55:1518-1522. 10.3109/10428194.2013.850685

4. Hafez HA, Soliaman RM, Bilal D, Hashem M, Shalaby LM: Early deaths in pediatric acute leukemia: a major challenge in developing countries. J Pediatr Hematol Oncol. 2019, 41:261-266. 10.1097/MPH.0000000000001408

5. O'Connor D, Bate J, Wade R, et al.: Infection-related mortality in children with acute lymphoblastic leukemia: an analysis of infectious deaths on UKALL2003. Blood. 2014, 124:1056-1061. 10.1182/blood-201403-560847

6. Fadoo Z, Nisar I, Yousuf F, et al.: Clinical features and induction outcome of childhood acute lymphoblastic leukemia in a lower/middle income population: a multi-institutional report from Pakistan. Pediatr Blood Cancer. 2015, 62:1700-1708. 10.1002/pbc.25583

7. Maaz A, Badar F, Mahmood MT, Al Nassir I: High infection related mortality in Pakistani children with acute lymphoblastic leukemia during remission induction chemotherapy: review of data from a single institution. J Cancer Allied Spec. 2016, 2:1-7. 10.37029/jcas.v2i4.94 
8. Asim M, Zaidi A, Ghafoor T, Qureshi Y: Death analysis of childhood acute lymphoblastic leukaemia; experience at Shaukat Khanum Memorial Cancer Hospital and Research Centre, Pakistan. J Pak Med Assoc. 2011, 61:666-670.

9. Howard SC, Pedrosa M, Lins M, Pedrosa A, Pui CH, Ribeiro RC, Pedrosa F: Establishment of a pediatric oncology program and outcomes of childhood acute lymphoblastic leukemia in a resource-poor area. JAMA. 2004, 291:2471-2475. 10.1001/jama.291.20.2471

10. Goulden NJ, Kirkwood AA, Moppett J, et al.: UKALL 2011: randomised trial investigating a short induction dexamethasone schedule for children and young adults with acute lymphoblastic leukaemia. Blood. 2017, 130:141. 10.1182/blood.V130.Suppl_1.141.141

11. Marwaha RK, Kulkarni KP, Bansal D, Trehan A: Pattern of mortality in childhood acute lymphoblastic leukemia: experience from a single center in northern India. J Pediatr Hematol Oncol. 2010, 32:366-369. 10.1097/MPH.0b013e3181e0d036

12. Viana SS, de Lima LM, do Nascimento JB, et al.: Secular trends and predictors of mortality in acute lymphoblastic leukemia for children of low socioeconomic level in Northeast Brazil. Leuk Res. 2015, 39:1060-1065. 10.1016/j.leukres.2015.07.007

13. Khan M, Shahzad M, Riaz S, et al.: Induction related mortality and morbidity in childhood acute lymphoblastic leukemia patients treated at tertiary care centre. SIOP. 2014, 61:260.

14. Rubnitz JE, Lensing S, Zhou Y, Sandlund JT, Razzouk BI, Ribeiro RC, Pui CH: Death during induction therapy and first remission of acute leukemia in childhood: the St. Jude experience. Cancer. 2004, 101:1677-1684. 10.1002/cncr.20532

15. Greenberg D, Moser A, Yagupsky P, Peled N, Hofman Y, Kapelushnik J, Leibovitz E: Microbiological spectrum and susceptibility patterns of pathogens causing bacteraemia in paediatric febrile neutropenic oncology patients: comparison between two consecutive time periods with use of different antibiotic treatment protocols. Int J Antimicrob Agents. 2005, 25:469-473. 10.1016/j.ijantimicag.2005.01.020

16. Tüfekçi Ö, Yılmaz Bengoa Ş, Demir Yenigürbüz F, Şimşek E, Karapınar TH, İrken G, Ören H: Management of invasive fungal infections in pediatric acute leukemia and the appropriate time for restarting chemotherapy. Turk J Haematol. 2015, 32:329-337. 10.4274/tjh.2014.0035

17. Baytan B, Güneş AM, Çelebi S, Günay Ü: Invasive fungal diseases in children with hematologic disorders . Turk J Hematol. 2009, 26:190-196.

18. Marr KA, Patterson T, Denning D: Aspergillosis. Pathogenesis, clinical manifestations, and therapy . Infect Dis Clin North Am. 2002, 16:875-894. 10.1016/s0891-5520(02)00035-1 